\title{
A Prospective study of laser treatment of port wine stain associated with gold nanorods
}

\begin{abstract}
Laser therapy based on the selective photothermolysis has been regarded as the most effective treatment strategy for Port-Wine Stain (PWS) caused by the expansion of dermal capillaries. A long-pulsed Neodymium: Yttrium Aluminum Garnet (Nd:YAG) laser has great potential for deeply situated PWS. However, the weak absorption of blood to Nd:YAG laser limits the laser treatment efficacy. Gold nanorods, which can convert the absorbed nearinfrared light into localized heat, have shown potential for photo-activated cancer therapy. Owing to their unique surface plasmon resonance and photothermal conversion effect, gold nanorods may be one of possible strategies for improving laser treatment efficacy of PWS by enhancing blood absorption to Nd:YAG laser. In this study, an experimental study was carried out to compare laser-induced thermal response of blood vessels in vivo animal model before and after injection of gold nanorods. The results demonstrated that the obvious thermal response of blood vessels to Nd:YAG can be obtained with lower laser energy fluence and less pulse number under the assistance of gold NRs, suggesting that Nd:YAG laser treatment combined with gold NRs may be a novel treatment strategy for PWS.
\end{abstract}

Volume 5 Issue 3 - 2017

\author{
Linzhuang Xing,' Bin Chen,' Dong Li,' \\ Wenjuan Wu,' Guoxiang Wang ${ }^{1,2}$ \\ 'Xi'an Jiaotong University, China \\ 2Department of Mechanical Engineering, University of Akron, \\ USA
}

\begin{abstract}
Correspondence: Bin Chen, State Key Laboratory of Multiphase Flow in Power Engineering, $\mathrm{Xi} \square$ an Jiaotong University, Xi $\square$ an, Shaanxi 710049, China,
\end{abstract} Email chenbin@mail.xjtu.edu.cn

Received: March 25, 2017 | Published: April II, 2017

\section{Introduction}

Port-Wine Stain (PWS) are congenital vascular lesions resulted from the expansion of capillaries and postcapillary venules.$^{1,2}$ Nowadays, laser treatment has remained the gold standard treatment for PWS, represented by pulsed dye laser with a wavelength of 585 or $595 \mathrm{~nm}$ and Neodymium: Yttrium Aluminum Garnet (Nd:YAG) laser with a wavelength of $1064 \mathrm{~nm} .{ }^{3} \mathrm{Nd}$ :YAG laser has great potential for treating deeper buried vascular lesions due to the advantages of lower absorption of melanin and deeper penetration into tissue., ${ }^{4,5}$ However, this treatment efficacy is limited by weak absorption of blood to Nd:YAG laser.

Gold nanorods (NRs) are rod-shaped gold nanoparticles with the characteristics of biocompatibility, surface plasmon resonance, and high photothermal efficiency. ${ }^{6}$ leading to the major applications of gold NRs in photoacoustic imaging, drug delivery and photothermal therapy of cancer cells.$^{7,8}$ Gold NRs exhibit two surface plasmon bands. One is located in the near- infrared region ranging from 600 to $1200 \mathrm{~nm}$, which is sensitive to the aspect ratio (length/width) of gold NRs. The intense absorption coefficient of this band results in high photothermal efficiency by converting absorbed near-infrared light to localized heat. Giving their unique biocompatibility, distinct surface plasmon band, and photothermal conversion effect, gold NRs has potential for enhancing blood absorption of blood to specific laser and destroying the vascular lesions.

In the present communication, an experimental study was carried out about laser-induced thermal response of blood vessels in vivo animal model after intravenous injection of gold NRs to investigate the potential use of gold NRs in the treatment of PWS.

\section{Materials and Methods}

Gold NRs were prepared by popular seeded growth method developed by Ye et al. ${ }^{9,10}$ And modified through replacing cytotoxic cetyltrimethylammonium bromide (CTAB) used as surfactant in the preparation of gold NRs by thiol-terminated methoxypolyethylene glycol (mPEG-SH) to reduce its cytotoxicity. The detailed preparation procedure can be found in the previous publication..$^{11}$ The absorption spectra and transmission electron microscopy (TEM) images were obtained using spectrometer (DH-2000-BAL, Ocean Optics, Germany) and JEOL 2100 (Japan) microscope operated at $300 \mathrm{kV}$, respectively. A dorsal skin-fold chamber (DSC) model was prepared according to the report of Papenfuss et al. [12] to simulate the vasculature of PWS. Thereafter, $0.44 \mathrm{mg}$ of gold NRs were intravenously injected into mice from tail veil, and laser irradiation was fired. Prior to and after laser irradiation, the images of blood vessels were recorded by a microscopy camera with 4 times magnification (BX41, Olympus Corp., Japan).

\section{Results and Discussion}

Figure 1 presented the absorption spectra and TEM images of gold NRs. CTAB-coated gold NRs had two absorption bands corresponding to the longitudinal absorption band at $1053 \mathrm{~nm}$ and transverse absorption band at $514 \mathrm{~nm}$. After modification using PEG, gold NRs showed the same two absorption bands at the identical wavelengths, suggesting that no aggregates occurred in the preparation procedure. In this study, the average length and width of the PEG-modified gold NRs were $79.5 \pm 10.5 \mathrm{~nm}$ and $13.5 \pm 0.9 \mathrm{~nm}$, respectively, with the aspect ratio of 5.89 .

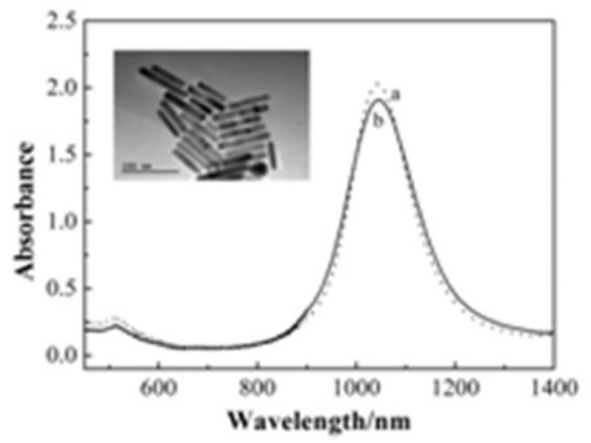

Figure I Absorption spectra of CTAB-modified gold NRs (a) and PEGmodified gold NRs (b). The inset shows a TEM image of PEG-modified gold NRs. 
Figure 2 displayed the thermal response of blood vessels as a control experiment before and immediately after $\mathrm{Nd}$ :YAG laser irradiation. The pulse duration of Nd:YAG laser was $0.3 \mathrm{~ms}$ with the spot diameter of $2 \mathrm{~mm}$, and the pulse repetition rate was $10 \mathrm{~Hz}$. We can find that no obvious changes of blood vessel were observed after laser irradiation by Nd:YAG laser with laser energy fluence of $36 \mathrm{~J} /$ $\mathrm{cm} 2$ for 20 laser pulses.
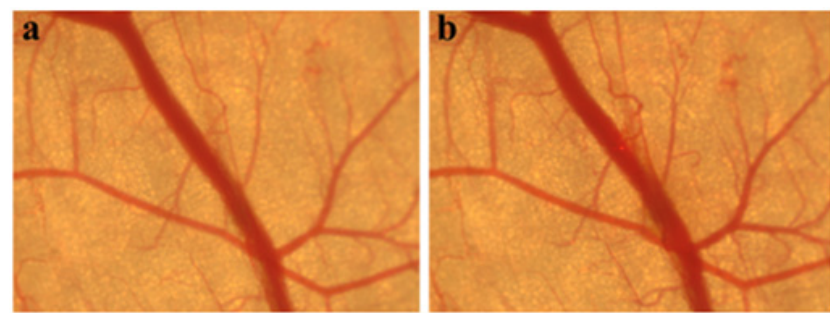

Figure 2 Blood vessels of mouse as a control experiment before (a) and immediately after laser irradiation with laser energy fluence of $36 \mathrm{~J} / \mathrm{cm}^{2}$ for 20 laser pulses (b).

Figure 3 exemplified the thermal response of blood vessels injected with PEG-modified gold NRs before and after laser irradiation with same pulse duration, spot diameter, and pulse repetition rate. After exposure to Nd:YAG laser with laser energy fluence of $27 \mathrm{~J} / \mathrm{cm} 2$ for 10 laser pulses, the constriction of blood vessels was observed. Compared to thermal damage of blood vessels without injection of gold NRs (Figure 2), obvious thermal response of blood vessels to Nd:YAG laser was obtained with lower energy fluence and less laser pulse. After intravenous administration of gold NRs, the blood absorption to Nd:YAG laser enhanced greatly, yielding lower laser energy fluence and less pulse number required for the obvious thermal damage. These observations indicated that Nd:YAG laser treatment of PWS with lower laser energy fluence may be obtained via combining gold NRs.
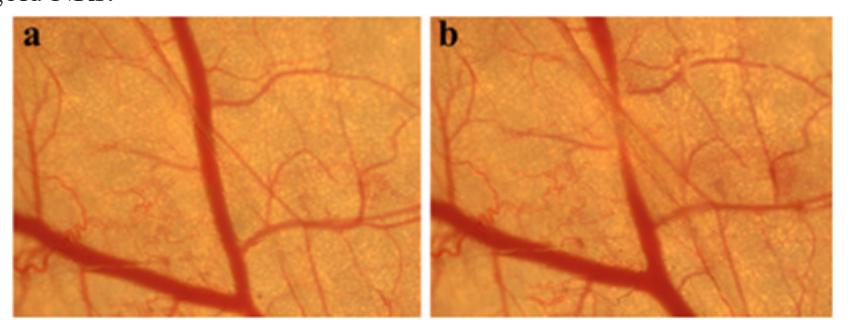

Figure 3 Blood vessels of mouse injected with PEG-modified gold NRs before (a) and immediately after laser irradiation with laser energy fluence of $27 \mathrm{~J} / \mathrm{cm}^{2}$ for 10 laser pulses (b).

\section{Conclusion}

In the present communication, an experimental study was performed to compare the laser energy fluence and pulse number required for the obvious thermal response of blood vessels to Nd:YAG laser with and without intravenous injection of gold NRs. The gold NRs had a longitudinal absorption band at $1053 \mathrm{~nm}$ with the aspect ratio of 5.89, which is close to that of the incident Nd:YAG. The blood absorbance increased significantly under the assistance of gold NRs, suggesting that obvious thermal response of blood vessels to Nd:YAG can be obtained with lower laser energy fluence and less pulse number, which can avoid thermal damage of epidermis and reduce the side effect in clinical. This communication is a prospective study about laser treatment of PWS associated with gold NRs, and much more future work will be done for the clinical application.

\section{Acknowledgements}

The work was jointly supported by the National Natural Science Foundation of China (51336006) and the Fundamental Research Funds for the Central Universities.

\section{Conflict of interest}

None.

\section{References}

1. Kelly KM, Kimel S, Smith T et al. Combined photodynamic and photothermal induced injury enhances damage to in vivo model blood vessels. Lasers Surg Med. 2004;34(5):407-413.

2. Li D, Farshidi D, Wang GX et al. A comparison of microvascular responses to visible and near-infrared lasers. Lasers Surg Med. 2014;46(6):479-487.

3. Alster TS, Tanzi EL Combined 595-nm and 1,064-nm laser irradiation of recalcitrant and hypertrophic port-wine stains in children and adults. Dermatol Surg. 2009;35(6):914-919.

4. Izikson L, Nelson JS, Anderson RR Treatment of hypertrophic and resistant port wine stains with a $755 \mathrm{~nm}$ laser: a case series of 20 patients. Lasers Surg Med. 2009;41(6):427-432.

5. Sherwood KA, Murray S, Kurban AK et al. Effect of wavelength on cutaneous pigment using pulsed irradiation. $J$ Investig Dermatol. 1989;92(5):717-720.

6. Huang XH, Jain PK, El Sayed IH et al. Plasmonic photothermal therapy (PPTT) using gold nanoparticles. Lasesr Med Sci. 2008;23(3):217-228.

7. Lakhani PM, Rompicharla SVK, Ghosh B et al. An overview of synthetic strategies and current applications of gold nanorods in cancer treatment. Nanotechnology. 2015;26(43):432001.

8. Heidari Z, Salouti M, Sariri R Breast cancer photothermal therapy based on gold nanorods targeted by covalently-coupled bombesin peptide. Nanotechnology. 2015;26(19):195101.

9. Ye $\mathrm{XC}$, Zheng $\mathrm{C}$, Chen $\mathrm{J}$ et al. Using binary surfactant mixtures to simultaneously improve the dimensional tunability and monodispersity in the seeded growth of gold nanorods. Nano Lett. 2013;13(2):765-771.

10. Ye XC, Jin LH, Caglayan H et al. Improved size-tunable synthesis of monodisperse gold nanorods through the use of aromatic additives. ACS Nano. 2012;6(3):2804-2817.

11. Xing LZ, Chen B, Li D, Ma J et al. Nd:YAG laser-induced morphology change and photothermal conversion of gold nanorods with potential application in the treatment of port-wine stain. Lasers Med Sci. 2017;32(3):629-640.

12. Papenfuss HD, Gross JF, Intaglietta M et al. Transparent access chamber for the rat dorsal skin fold. Microvasc Res. 1979;18(3):311-318. 